\title{
AQP5: A novel biomarker that predicts poor clinical outcome in colorectal cancer
}

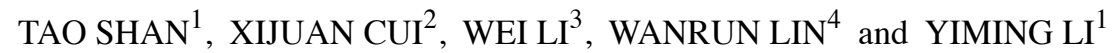 \\ ${ }^{1}$ Department of General Surgery, The Second Affiliated Hospital of Medical College, Xi'an Jiaotong University, \\ Xi'an, Shaanxi 710004; ${ }^{2}$ Department of General Surgery, The First Affiliated Hospital of Medical College, Xi'an Jiaotong \\ University, Xi'an, Shaanxi 710061; ${ }^{3}$ Graduate School of The Fourth Military Medical University, Xi'an, Shaanxi 710032; \\ ${ }^{4}$ Department of Pathology, Provincial Hospital Affiliated to Shandong University, Jinan, Shandong 250021, P.R. China
}

Received April 5, 2014; Accepted June 12, 2014

DOI: $10.3892 / o r .2014 .3377$

\begin{abstract}
The aquaporins (AQPs) are water channel proteins that exhibit several properties related to tumor development. However, the expression and clinical significance of AQP5 in colorectal cancer, particularly the correlation with circulating tumor cells (CTCs), has not been elucidated. The aim of the present study was to determine whether or not the expression of AQP5 is a strong prognostic biomarker for colorectal cancer. The results showed that of 45 tumor specimens, 14 (31.1\%) had high levels of expression of AQP5, 29 (64.4\%) exhibited a moderate (intermediate) level of staining, and $2(4.4 \%)$ had an absence of AQP5 staining. AQP5 was only occasionally detected in para-neoplastic [3/45 (6.67\%)] and normal tissues [3/45 (6.67\%)]. AQP5 protein overexpression frequently accompanied gene amplification detection with fluorescence in situ hybridization (FISH). Moreover, AQP5 expression in colorectal cancer cells was upregulated compared to normal colon cells. AQP5 overexpression was also associated with TNM stage $(\mathrm{P}=0.002)$, lymph node metastasis $(\mathrm{P}=0.016)$, and distant metastasis $(\mathrm{P}=0.000)$. The relationships between age, gender, histologic grade and tumor size with expression of AQP5 were not significant $(\mathrm{P}>0.05)$. A positive correlation between the number of CTCs and AQP5 expression $(\mathrm{P}<0.05)$ was demonstrated. In addition, patients who did not express AQP5 had a superior cumulative survival rate compared to patients with AQP5 positivity. AQP5 may be used as a novel biomarker for colorectal cancer aggressiveness and metastasis, but it does not reflect drug resistance.
\end{abstract}

Correspondence to: Dr Tao Shan, Department of General Surgery, The Second Affiliated Hospital of Medical College, Xi'an Jiaotong University, 157 Xiwu Road, Xi'an, Shaanxi 710004, P.R. China E-mail: shantao820304@163.com

Key words: colorectal cancer, aquaporin 5, prognosis, circulating tumor cells, fluorescence in situ hybridization

\section{Introduction}

Colorectal cancer remains the second most common cause of cancer-related mortality in the United States. Of the patients with colorectal cancer who die, most succumb with a significant burden of metastatic disease (1). Colorectal cancer is an aggressive and intractable human malignant tumor, despite the advances in the diagnosis and treatment (2). Therefore, it is highly important to seek novel targets for therapeutic intervention.

Recently, increasingly more studies have shown that tumor growth, development, invasion and metastasis depend on the tumor microenvironment and tumor metabolism (3). Water molecules play a significant role in the progression of malignant epithelial tumors, an understanding of which is thus important in anticancer treatment strategy (4). Aquaporins (AQPs) are a family of membrane water channels that are required for the transport of water through many secretory and absorptive epithelia. Some subtypes of AQPs are also involved in the transport of other molecules, such as glycerol and urea. There are currently 13 AQP members which have been identified in mammals. Among them, AQP0, AQP1, AQP2, AQP4, AQP5, AQP6 and AQP8 are primarily water-selective, whereas AQP3, AQP7, AQP9, AQP10 and AQP12 also transport glycerol and other small solutes (5). An altered expression of AQPs has been revealed in several types of tumors based upon their specific tissue localization. Expression of AQP1 is frequently associated with brain tumors (6). In studies involving AQP3-null mice, AQP3 gene deletion induced resistance to carcinogeninduced skin tumors. Glycerol transport through AQP3 also contributes to the generation of ATP for cell proliferation and tumorigenesis (7). AQP5 is widely overexpressed in pancreatic cancer and appears to be involved in cell proliferation (8).

In particular, AQP5 expression in colon cancer tissues is associated with metastasis, suggesting that AQP5 overexpression plays a role in cancer progression (9). Ras signal transduction has been suggested to enhance cell proliferation in AQP5-overexpressed NIH3T3 cells (10). Moreover, a molecular study revealed that AQP5 binds to the SH3 domains of c-Src, a non-receptor cytoplasmic tyrosine kinase associated with invasive and metastatic phenotypes in various tumors (11). However, the expression and clinical significance of AQP5 in 
colorectal cancer, particularly the correlation with circulating tumor cells (CTCs), has not been elucidated. To evaluate the potential of AQP5 as a novel prognostic marker of colorectal cancer, we used immunohistochemical, RT-PCR, real-time PCR, western blotting and fluorescence in situ hybridization (FISH) methods to detect the expression and amplification of the AQP5 gene in clinical samples of colorectal cancer, and immunofluorescence in situ hybridization (imFISH) staining. We then analyzed the correlations between the expression of AQP5 and clinicopathologic features, CTCs and prognosis of colorectal cancer.

\section{Materials and methods}

Patient specimens. From January 2008 to December 2013 , colorectal cancer tissues (including adequately sized tumor tissue samples and tissue samples obtained from areas within $2.0 \mathrm{~cm}$ around the tumor) were obtained from 45 patients with colorectal cancer at the Department of General Surgery, Second Affiliated Hospital of Xi'an Jiaotong University. Samples were fixed with $4 \%$ formalin for histological studies. Of the 45 patients, 24 were male and 21 were female. Median age at the time of surgery was 58.3 years (range, $40-78$ years). The histological type in all 45 patients was colorectal adenocarcinoma. Tumor stage and histopathological grading were recorded according to the classification of the International Union Against Cancer. There were 3 stage I, 11 stage II, 27 stage III, and 4 stage IV tumors. Histological grades for the patients were as follows: 7 patients grade I, 20 grade II and 18 grade III. All patients were followed up and the median duration of follow-up was 23 months (5-53 months). All the studies were approved by the Human Subjects Committee of Xi'an Jiaotong University, China. Consent forms signed by all patients recruited in this study were approved by the Ethics Review Committee of the Human Subjects Committee of the Xi'an Jiaotong University, China.

Immunohistochemistry. AQP5 protein was detected immunohistochemically using a standardized streptavidin-peroxidase (SP) method. Tissue sections $(4 \mu \mathrm{m})$ were incubated overnight with primary antibody at a proper concentration. The next day, the slides were incubated for $30 \mathrm{~min}$ with biotinylated goat anti-rabbit IgG, followed by incubation with peroxidaseconjugated streptavidin for $20 \mathrm{~min}$ at room temperature. Color was developed using $0.02 \%$ 3, 3'-diaminobenzidine (DAB) in $50 \mathrm{mM}$ Tris- $\mathrm{HCl}$ buffer ( $\mathrm{pH} 7.6$ ) for 5-7 min. Finally, the sections were counterstained with hematoxylin, rinsed with water, dehydrated, cleared and coverslipped. Negative controls for immunostaining replaced the primary antibody with nonimmune goat or rabbit serum. The number of stained cells per 1,000 was determined under a microscope (Olympus Optical, Tokyo, Japan) in three visual fields, at a x400 magnification. When the total number of cells observed under the microscope was $<1,000$, all cells were counted. The staining was scored semiquantitatively as negative ( 0 , no staining); moderate (1, either diffuse weak staining or strong staining in $<30 \%$ of cells per core); or strong ( 2 , strong staining of $\geq 30 \%$ of the cells). The antibodies against AQP5 and $\beta$-actin were purchased from Santa Cruz Biotechnology (Santa Cruz, CA, USA).
RT-PCR and real-time PCR. Total RNA was extracted from colorectal tumor, peri-tumor and normal tissue using TRIzol reagent (Gibco-BRL). First-strand cDNA was synthesized from $2 \mu \mathrm{g}$ of total RNA using the RevertAid Kit (Fermentas MBI, Amherst, NY, USA). The PCR primer sets were designed: i) for AQP5, forward, TGACGAGGACTGGGAGG AGC-3' and reverse, 5'-GGCGGCATTCAATGAACCA-3'; ii) for $\beta$-actin, forward, 5'-ATCGTGCGTGACATTAAGGAG AAG-3' and reverse, 5'-AGGAAGGAAGGCTGGAAGA GTG-3'. The PCR conditions included an initial denaturation step for $5 \mathrm{~min}$ at $95^{\circ} \mathrm{C}$ followed by 22 cycles of amplification: $30 \mathrm{sec}$ at $95^{\circ} \mathrm{C}, 30 \mathrm{sec}$ at $57^{\circ} \mathrm{C}$ and $30 \mathrm{sec}$ at $72^{\circ} \mathrm{C}$. After the last cycle, a final extension was performed at $72^{\circ} \mathrm{C}$ for $10 \mathrm{~min}$. The housekeeping gene $\beta$-actin was used as an internal control.

Real-time quantitative PCR was carried out with Platinum SYBR-Green qPCR SuperMix UDG (Invitrogen, Carlsbad, CA, USA) using the Rotor-Gene RG-3000 (Corbett Research, Doncaster Victoria, Australia). For each amplicon, the amount of AQP5 and $\beta$-actin was determined from a standard curve generated by serial dilution. Prior to amplification, the samples were incubated at $95^{\circ} \mathrm{C}$ for $10 \mathrm{~min}$, and each amplification cycle consisted of denaturation for $45 \mathrm{sec}$ at $95^{\circ} \mathrm{C}$, annealing for $30 \mathrm{sec}$ at $57^{\circ} \mathrm{C}$ and extension for $30 \mathrm{sec}$ at $73^{\circ} \mathrm{C}$. The amount of target genes in the cDNA samples was calculated based on the threshold cycle $(\mathrm{Ct})$. The PCR signals were quantitated by densitometric analysis using Quantity One ${ }^{\circledR}$ analysis software.

Western blotting. Colorectal tumor tissues were minced and incubated on ice for $30 \mathrm{~min}$ in $0.5 \mathrm{ml}$ of ice-cold whole-cell lysate buffer (10\% NP-40, $5 \mathrm{M} \mathrm{NaCl}, 1 \mathrm{M}$ HEPES, $0.1 \mathrm{M}$ EGTA, 0.5 M EDTA, 0.1 M PMSF, 0.2 M sodium orthovanadate, $1 \mathrm{M} \mathrm{NaF}, 2 \mu \mathrm{g} / \mathrm{ml}$ aprotinin, $2 \mu \mathrm{g} / \mathrm{ml}$ leupeptin). The minced tissue was homogenized using a Dounce homogenizer and centrifuged at $16,000 \times \mathrm{g}$ at $4^{\circ} \mathrm{C}$ for $10 \mathrm{~min}$. The protein was separated by $10 \%$ SDS-PAGE and electro-transferred onto nitrocellulose membranes. After being blocked with $5 \%$ non-fat milk in TBST $(20 \mathrm{mM}$ Tris, $150 \mathrm{mM} \mathrm{NaCl}$, $0.2 \%$ Tween-20, $\mathrm{pH} 7.6$ ), the membranes were incubated with primary antibodies at $4^{\circ} \mathrm{C}$ overnight, followed by $1: 2,000$ horseradish peroxidase (HRP)-conjugated secondary antibody for $2 \mathrm{~h}$. Immunoreactive bands were visualized using an enhanced chemiluminescence kit (Amersham Pharmacia Biotech, Piscataway, NJ, USA). The western blotting signals were quantitated by densitometric analysis using Total Lab Nonlinear Dynamic Image ${ }^{\circledR}$ analysis software (Nonlinear, USA). Final histogram results = Target gene absolute value/ $\beta$-actin absolute value.

Isolation of normal colon cells and culture of colorectal cancer cells. Normal colon cells were isolated from colorectal site in normal colorectal specimens. Briefly, each specimen was collected and transferred to the laboratory. After several washings with sterile phosphate-buffered saline (PBS), 1-cm ${ }^{2}$ pieces of tissues were placed into the wells of culture flasks. Once the tissue appeared to attach flasks (5-6 h), Dulbecco's modified Eagle's medium (DMEM) containing 10\% FBS was added gently to the tissue pieces. Specimens were inspected daily and the medium was exchanged after $24 \mathrm{~h}$ for the first time and every third day thereafter. Tissue samples were then removed from the cultures and cells were transferred to larger 
tissue culture vessels once they had reached $70 \%$ confluence, after approximately 2 weeks.

The COLO 205 and SW480 colorectal cancer cell lines (the American Tissue Type Collection, USA) were maintained in DMEM, (Gibco-BRL) supplemented with penicillin $(100 \mathrm{U} / \mathrm{ml})$, streptomycin $(100 \mu \mathrm{g} / \mathrm{ml}), 0.1 \mathrm{mM}$ non-essential amino acids, $0.2 \mathrm{mM}$ glutamine, $1 \mathrm{mM}$ pyruvate, and $10 \%$ heat-inactivated fetal bovine serum (FBS) and incubated in a $5 \% \mathrm{CO}_{2}$ humidified atmosphere at $37^{\circ} \mathrm{C}$.

Immunofluorescence assay. Exponentially growing cells were seeded on $25-\mathrm{mm}$ square glass cover slips placed in $35-\mathrm{mm}$ diameter culture dishes. The cells were fixed with $4 \%$ formaldehyde for $5 \mathrm{~min}$, permeabilized with $0.2 \%$ solution of Triton X-100 in PBS, and blocked with $2 \%$ bovine serum albumin-PBS $30 \mathrm{~min}$. Slides were incubated with anti-AQP5 for overnight. Fluorescent imaging was obtained with a confocal laser scanning microscope (Carl Zeiss Micro Imaging).

FISH. AQP5 gene amplification was detected with dual-color FISH using a Passvision AQP5 DNA probe kit (Vysis Inc., Downers Grove, IL, USA), according to the manufacturer's instructions. Tissue sections ( $4-\mu \mathrm{m}$ thick) were baked overnight at $56^{\circ} \mathrm{C}$, and were handled with deparaffinization, enzyme digestion and fixation. The slides were then denatured in $70 \%$ formamide/2X standard saline citrate (SSC), at $72^{\circ} \mathrm{C}$ for $5 \mathrm{~min}$. After a buffer wash, $10 \mu \mathrm{l}$ of a mixture of two directly labelled probes (AQP5 specific sequence probe) were added to the tissue sections and hybridization was carried out at $37^{\circ} \mathrm{C}$ for 14-18 $\mathrm{h}$. The slides were then washed in a post-hybridization wash at $72^{\circ} \mathrm{C}$, counterstained with DAPI, mounted and stored in dark before signal enumeration. AQP5-spectrum red probe contains a DNA sequence specific for the AQP5 gene. Chromosome enumeration probe 17 (CEP17)/spectrum green probe containing $\alpha$-satellite DNA that hybridizes to the D17Z1 locus (centromere region of chromosome 17) was used as a control. The slides were observed under a fluorescence microscope equipped with a digital camera (DP50; Olympus, Tokyo, Japan). For each specimen, gene amplification was scored when a minimum of 20 cancer cell nuclei exhibited AQP5/CEP17 ratio $\geq 2$, or when AQP5 signal cluster was observed.

Blood sampling and enrichment of CTCs. Peripheral blood $(7.5 \mathrm{ml})$ collected in a BD Vacutainer tube (Becton, Dickinson and Co., Franklin Lakes, NJ, USA) was washed with PBS. In order to avoid epithelial cell contamination during venipuncture, all samples were collected after discarding the first $2 \mathrm{ml}$ blood. Red blood cells (RBCs) were mixed with $45 \mathrm{ml}$ lysis buffer $\left(155 \mathrm{mM} \mathrm{NH}_{4} \mathrm{Cl}, 10 \mathrm{mM} \mathrm{KHCO} 3,0.1 \mathrm{mM}\right.$ EDTA), followed by rotation for $8 \mathrm{~min}$ and centrifugation (600 x g for $5 \mathrm{~min}$ ) to remove RBCs. Resulting cell pellet was resuspended in PBS and subsequently incubated with $0.5 \mathrm{ml}$ of antileukocyte surface marker CD45 monoclonal antibody coated magnetic beads for 30 min, followed by separation of magnetic beads using a magnetic stand (Promega, Madison, WI, USA). Supernatants were transferred into a new tube, and subsequently centrifuged at $800 \mathrm{x}$ g for $3 \mathrm{~min}$. Cell pellets were spotted on glass slides, followed by imFISH staining.
imFISH staining (CEP8-CD45-DAPI). Tumor cells were negative enriched by the immunomagnetic beads method, followed by identification with cytology analysis. FISH was performed using centromere DNA probes of chromosome 8 (yellow) (Vysis), and immunofluorescence assay was performed using anti-CD45 (red) (Santa Cruz, CA, USA). The slides were washed three times with TBS $(10 \mathrm{mM}$ Tris, $2.8 \mathrm{mM} \mathrm{KCl}$, $137 \mathrm{mM} \mathrm{NaCl}, \mathrm{pH}$ 7.4) containing $0.2 \%$ BSA for $3 \mathrm{~min}$, and subsequently rinsed with TBS once. Cells were mounted with mounting medium containing the nuclear dye DAPI. A blinded review of the fluorescent images by three technicians confirmed the identity of the CTCs from 3-color fluorescent images that were magnified $\mathrm{x} 400$. Evaluation criteria for CTC identification from fluorescent images included both CEP8 $\geq 3$ and CD45 (-) staining pattern overlying the DAPI staining of the nucleus.

Statistical analysis and patient outcome. Data were analyzed by the $\chi^{2}$ or two-sided Fisher's exact test, as appropriate. Pearson's correlation coefficient was used to measure the strength of the association among AQP5 gene amplification, enumeration of CTCs and AQP5 expression levels. Survival rate was calculated by the Kaplan-Meier method, and differences were examined by the log-rank test. Factors found to be significant were then selected for a stepwise Cox's multivariate proportional hazard model to determine their prognostic values. Gene SNP was analyzed using the software of pyrosequencing equipment (PyroMark Q24, Qiagen, Germany). $\mathrm{P}<0.05$ was considered to indicate a statistically significant difference. All statistical analyses were performed using SPSS $^{\circledR}$ ver. 13.0 statistical software (SPSS, Chicago, IL, USA).

\section{Results}

Protein expression and gene amplification of AQP5 in colorectal cancer. It is known that AQP5 is expressed on the membrane and in the cytoplasm of cells. To determine the expression status of AQP5 in colorectal cancer, we first used immunohistochemistry to evaluate patient specimens in the colorectal cancer, peri-tumor and normal groups. The results highlighted the difference observed in AQP5 immunostaining in the compartment (Fig. 1A); specifically, 14/45 (31.1\%) of the patients had strong AQP5 expression and 29/45 (64.4\%) of the patients had moderate AQP5 expression in the cancer group. AQP5 was only occasionally detected in the peri-tumor [3/45, $(6.67 \%)]$ and normal tissues [3/45, (6.67\%)].

Since FISH is a more accurate and sensitive assay, and is generally regarded as the gold standard for detecting gene amplification compared to gene expression using immunohistochemistry (12), we further detected AQP5 amplification status using FISH. Notably, strong expression of AQP5 had a positive correlation with AQP5 gene amplification $(r=0.712$, $\mathrm{P}=0.000$; Fig. 2B; Table I). In contrast, the expression of AQP5 was low or absent in peri-tumor and normal samples, while the AQP5 gene was not amplified.

Finally, we continued to determine the expression status of AQP5 in samples by RT-PCR and real-time PCR. The AQP5 mRNA level was significantly upregulated in tumor samples when compared to peri-tumor and normal tissues (Fig. 1C and D; P<0.05). Similarly, western blotting results 
A

AQP5

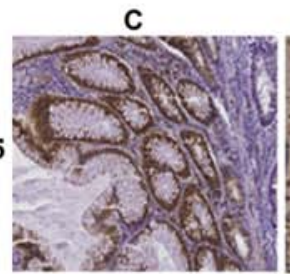

$\mathbf{P}$
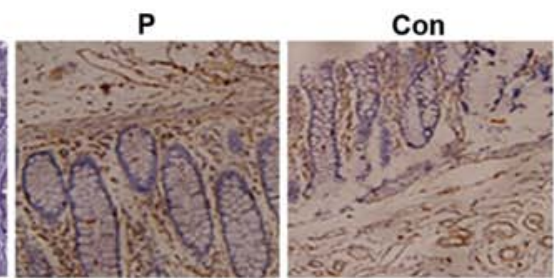

C
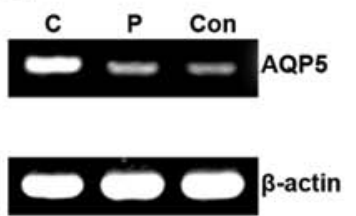

B

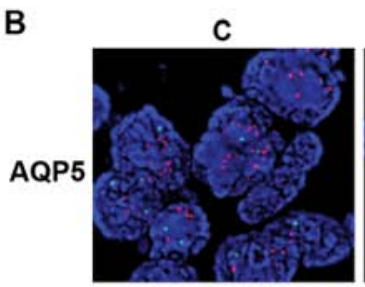

$\mathbf{P}$

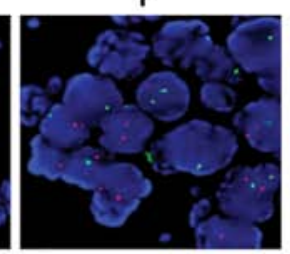

Con

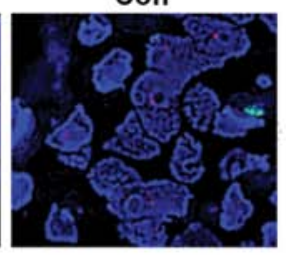

E

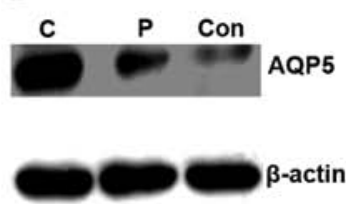

D

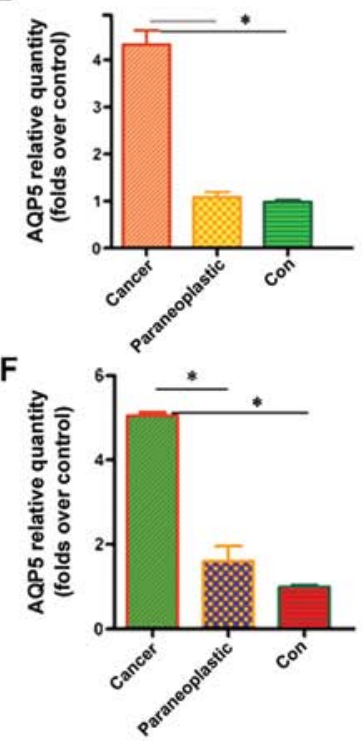

Figure 1. (A) Representative immunohistochemistry results of AQP5 expression in colorectal cancer. Paraffin sections were immunostained as described in Materials and methods. (B) AQP5 amplification status using FISH. (C and D) mRNA expression of AQP5 in samples by RT-PCR and real-time PCR. (E and F) Protein expression of AQP5 in samples by western blotting. Data from at least three independent experiments with duplicate determinations are expressed as the means \pm SEM. N, normal tissue; $P$, paracancer tissue; $C$, tumor tissue; magnification, $x 400$.
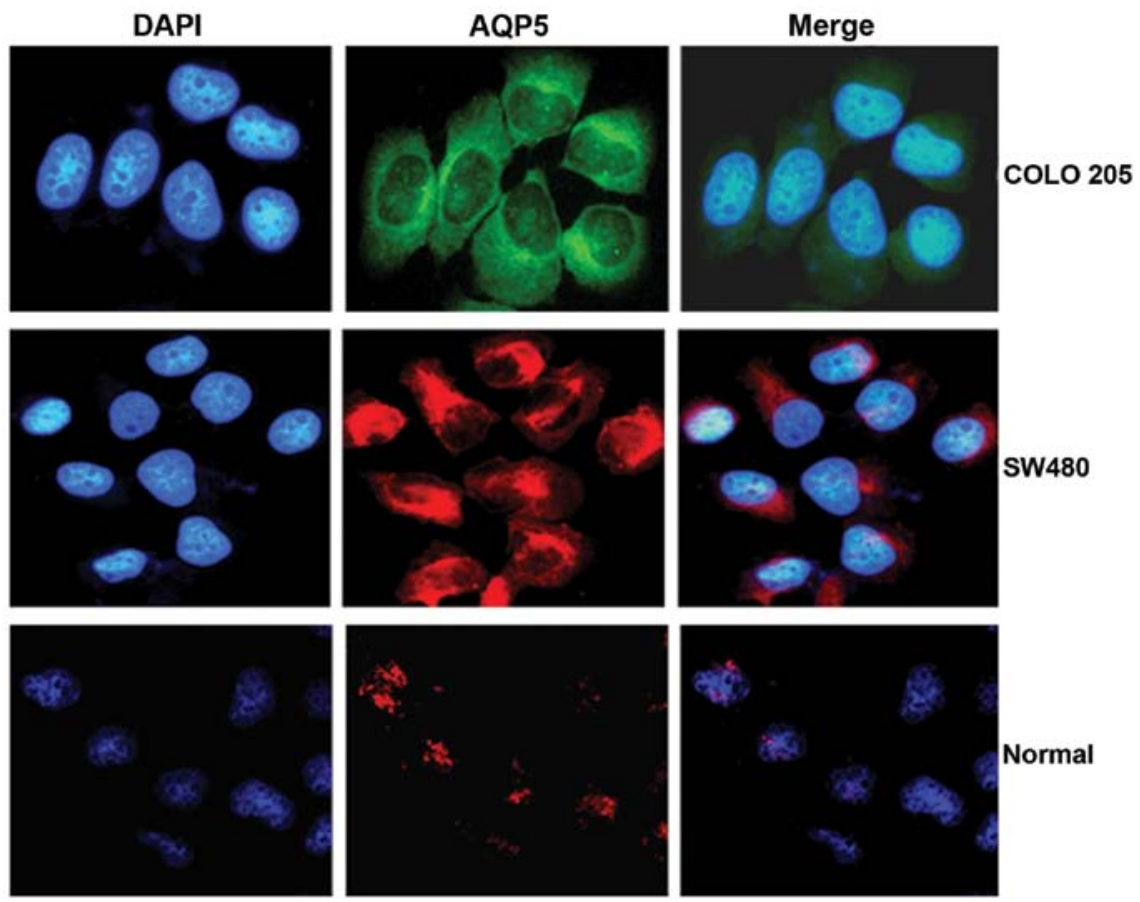

Figure 2. Immunodetection of AQP5 in colorectal cancer (COLO 205 and SW480) and normal cells (magnification, x200). AQP5 protein expression stained with CY3-labeled and FITC labeling-IgG antibody and analyzed by confocal microscopy.

showed that AQP5 protein was upregulated compared to peritumor and normal tissues (Fig. $1 \mathrm{E}$ and $\mathrm{F} ; \mathrm{P}<0.05$ ).

Expression of AQP5 in colorectal cancer and normal colon cells. We further determined AQP5 protein expression status in colorectal cancer cells (COLO 205 and SW480) and colon normal cells using an immunofluorescence assay and analyzed by confocal microscopy. The AQP5 fluorescence signal in colorectal cancer cells was increased compared to normal colon cells (Fig. 2). These findings further indicate that AQP5 is upregulated in colorectal cancer.

Relationship between AQP5 expression and clinicopathologic parameters. Table II summarizes the associations between AQP5 protein expression and clinicopathologic parameters in colorectal cancer. AQP5 was strongly expressed in 1/14 stage I-II cases $(7.1 \%)$, which was significantly lower than stage III $[33.3 \%,(9 / 27)]$ and stage IV [100.0\% (4/4)] cases 
Table I. Relationship between expression of AQP5 and amplification.

\begin{tabular}{lccccr}
\hline & & \multicolumn{3}{c}{ AQP5 } & \\
\cline { 3 - 5 } Gene & Case & $\begin{array}{c}0 \\
\mathrm{n}(\%)\end{array}$ & $\begin{array}{c}1 \\
\mathrm{n}(\%)\end{array}$ & $\begin{array}{c}2 \\
\mathrm{n}(\%)\end{array}$ & P-value \\
\hline Total & 45 & 2 & 29 & 14 & \\
AQP5 & & & & & $0.000^{\mathrm{a}}$ \\
Amplification & 15 & $0(0.0)$ & $2(6.9)$ & $13(92.9)$ & \\
Normal & 30 & $2(6.7)$ & $27(90.0)$ & $1(3.3)$ & \\
\hline
\end{tabular}

${ }^{\mathrm{a}} \mathrm{P}<0.05$; AQP, aquaporin.

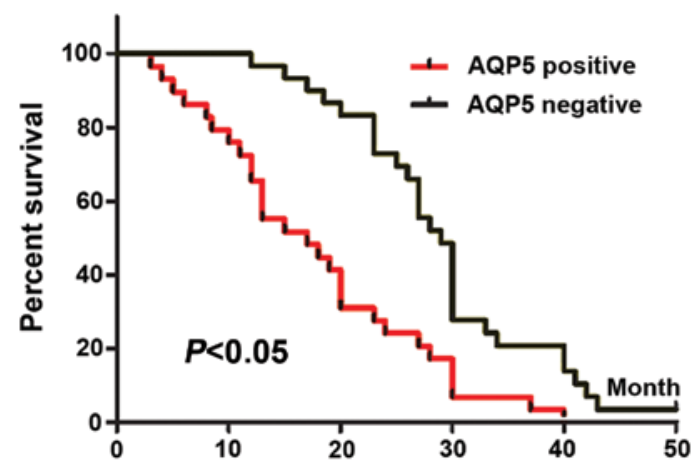

Figure 3. Kaplan-Meier analysis of the overall post-operative survival curves in colorectal cancer cases according to their immunohistochemical staining as positive or negative of AQP5 protein expression.

$(\mathrm{P}=0.002)$. AQP5 was also associated with lymph node $(\mathrm{P}=0.016)$ and distant metastases $(\mathrm{P}=0.000)$. We also determined the relationships between age, gender, histologic grade, and tumor size with expression of AQP5, however no significant relationships were observed $(\mathrm{P}>0.05)$.

Prognostic value of AQP5 in patients with colorectal cancer. To determine the prognostic value of AQP5 for colorectal cancer, we analyzed the cumulative survival of patients based on AQP5 status (Fig 3). AQP5 weak and no staining were merged as negative, while strong staining was regarded as positive. The cumulative survival rate in AQP5-negative patients $(\mathrm{n}=31)$ at 3 years was $24.3 \%$ (median time, 21.2 months). In contrast, the cumulative survival rate in AQP5-positive patients $(n=14)$ was $7.4 \%$ (median time, 7.7 months), a difference that was highly statistically significant $(\mathrm{P}<0.05)$.

Multivariate analysis revealed that lymph node metastasis $(\mathrm{P}=0.018)$ and TNM stage $(\mathrm{P}=0.031)$ were independent prognostic factors for overall survival in patients with colorectal cancer; tumor diameter and other clinical parameters were not independent prognostic factors.

Expression of AQP5 correlates with CTC enumeration. CTCs are tumor cells that are shed from the primary tumor into the circulation. The presence of CTCs in the peripheral blood of patients has long been associated with metastasis and poor survival, although some authorities debate the biologic
Table II. Association between AQP5 protein expression and clinicopathologic factors in colon cancer.

\begin{tabular}{|c|c|c|c|c|c|}
\hline \multirow[b]{2}{*}{ Variables } & \multirow[b]{2}{*}{ Case } & \multicolumn{3}{|c|}{ AQP5 } & \multirow[b]{2}{*}{ P-value } \\
\hline & & $\begin{array}{c}0 \\
\mathrm{n}(\%)\end{array}$ & $\begin{array}{c}1 \\
\mathrm{n}(\%)\end{array}$ & $\begin{array}{c}2 \\
\mathrm{n}(\%)\end{array}$ & \\
\hline Total & 45 & 2 & 29 & 14 & \\
\hline Age (years) & & & & & 0.848 \\
\hline$>60$ & 28 & $1(3.6)$ & $18(64.3)$ & $9(32.1)$ & \\
\hline$\leq 60$ & 17 & $1(5.9)$ & $11(64.7)$ & $5(29.4)$ & \\
\hline Gender & & & & & 1.000 \\
\hline Female & 21 & $1(4.8)$ & $14(66.7)$ & $7(33.3)$ & \\
\hline Male & 24 & $1(4.2)$ & $15(62.5)$ & $7(29.2)$ & \\
\hline Histologic grade & & & & & 0.913 \\
\hline I & 7 & $2(28.6)$ & $4(57.1)$ & $2(28.6)$ & \\
\hline II & 20 & $0(0.0)$ & $12(60.0)$ & $6(30.0)$ & \\
\hline III & 18 & $0(0.0)$ & $13(72.2)$ & $6(33.3)$ & \\
\hline Tumor size $(\mathrm{cm})$ & & & & & 0.263 \\
\hline$\leq 2$ & 15 & $1(6.7)$ & $10(66.7)$ & $4(26.7)$ & \\
\hline $2-5$ & 26 & $1(3.8)$ & $18(69.2)$ & $8(30.8)$ & \\
\hline$>5$ & 4 & $0(0.0)$ & $1(25.0)$ & $2(50.0)$ & \\
\hline $\begin{array}{l}\text { Lymph node } \\
\text { metastasis }\end{array}$ & & & & & 0.016 \\
\hline Negative & 17 & $2(11.8)$ & $8(47.0)$ & $3(17.6)$ & \\
\hline Positive & 28 & $0(0.0)$ & $19(67.9)$ & $11(39.3)$ & \\
\hline $\begin{array}{l}\text { Distant } \\
\text { metastasis }\end{array}$ & & & & & 0.000 \\
\hline Negative & 39 & $2(5.1)$ & $29(74.3)$ & $8(20.5)$ & \\
\hline Positive & 6 & $0(0.0)$ & $0(0.0)$ & $6(100.0)$ & \\
\hline TNM stage & & & & & 0.002 \\
\hline $\mathrm{I} / \mathrm{II}$ & 14 & $1(7.1)$ & $12(85.7)$ & $1(7.1)$ & \\
\hline III & 27 & $1(3.7)$ & 17 (62.9) & $9(33.3)$ & \\
\hline IV & 4 & $0(0.0)$ & $0(0.0)$ & $4(100.0)$ & \\
\hline
\end{tabular}

AQP5 staining score 0 (no staining), 1 (weak) were counted as negative and score 2 (strong) counted as positive; the two groups were compared using the $\chi^{2}$ test. AQP, aquaporin.

significance of CTCs due to tumor genomic instability and potential metastatic inefficiency. In view of the obvious clinical relevance, CTCs have been recently recommended by the American Society of Clinical Oncology as an acceptable cancer marker. To further determine whether or not AQP5 is an adverse prognostic biomarker, the correlation between AQP5 expression and the number of CTCs was analyzed. CTCs were detected in $78.57 \%$ (11/14) of colorectal cancer patients with strong AQP5 expression vs. $45.16 \%$ (14/31) with low and absent AQP5 expression ( $\mathrm{P}<0.05$; Fig. 4); Moreover, a significantly greater number of CTC enumeration was observed in patients with strong expression of AQP5 compared to patients with low and absent expression of AQP5 (19.5 2.0 in $7.5 \mathrm{ml}$ blood vs. $5.5 \pm 1.5 \mathrm{in} 7.5 \mathrm{ml}$ blood). In conclusion, AQP5 is positively correlated with the number of CTCs. 

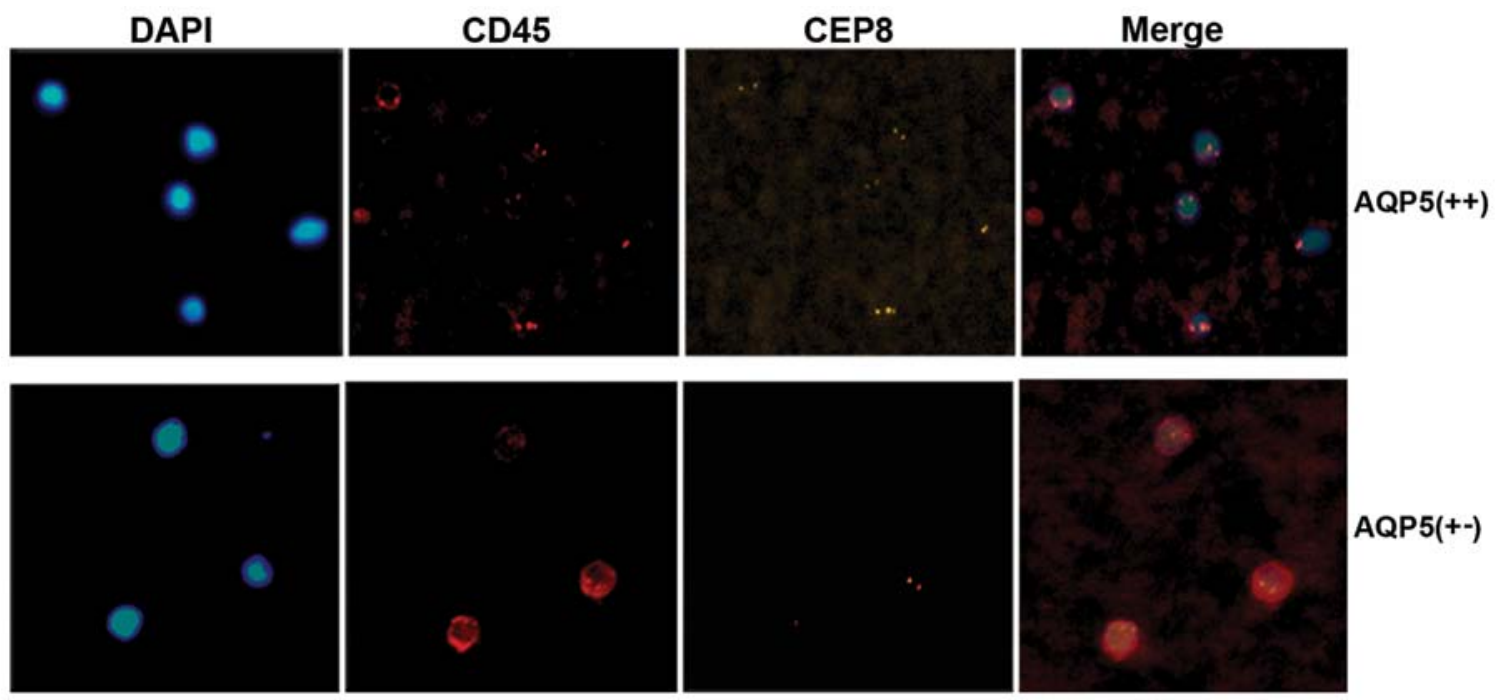

Figure 4. CTCs stained with anti-CD45-CEP8-DAPI in peripheral blood of colorectal cancer patients (magnification, $\mathrm{x} 400$ ).

\section{Discussion}

Colorectal cancer is characterized by early lymph node metastasis and poor prognosis (1). In the present study, we determined the clinical significance of AQP5 expression in colorectal cancer patients, and thereby better defined the potential role for AQP5 in novel therapeutic modalities. Our results revealed that AQP5 is upregulated in colorectal cancer compared with peri-tumor and normal tissues accompanied by AQP5 DNA amplification. Furthermore, AQP5 was closely correlated with advanced TNM stage and poor prognosis. Markedly, AQP5overexpressing cancer was shown to be prone to metastasis; clearly, there are more CTCs circulating in patients with AQP5 overexpression. These findings extend our understanding of the role of AQP5 as a diagnostic marker. To our knowledge, this is the first study to show AQP5 in colorectal cancer and its association with CTCs.

Aquaporins play a crucial role in maintaining water homeostasis and modulating a variety of physiologic and pathologic processes (13). Notably, the present study suggested that AQPs, including AQP5, are involved in tumorigenesis. AQP5, a 21-24 kDa protein, was initially described as the main structural protein in caveolae and was believed to be a key molecule involved in oncogenic transformation and malignant progression $(14,15)$. AQP5 plays an important regulatory role in several signaling pathways leading to cellular transformation, including those mediated by the Src family of tyrosine kinases, epidermal growth factor receptor, Wnt, and Erk1/2 (10,16,17). Recently, Lee et al (11) reported that AQP5 overexpression is significantly associated with lymph node involvement and a poorer prognosis in patients with breast cancer, suggesting the value of AQP5 as a prognostic marker in breast cancer. Huang et al (18) also verified that AQP5 promotes the proliferation and migration of human gastric carcinoma cells. Wang et al (19) performed a study to show that AQP5 was mainly expressed in colorectal carcinoma cells and barely expressed in paraneoplastic normal tissues. The clinical significance of AQP5 in colorectal carcinoma is unknown. Our results showed that AQP5 is mainly expressed in colorectal cancer cells and minimally expressed in peritumor and normal tissues. The expression patterns of AQP5 in colorectal tissues detected herein are consistent with the results of previous studies. Furthermore, we analyzed the correlations of AQP5 expression with the clinicopathologic features of colorectal cancer and showed that AQP5 expression is not significantly associated with the gender or age of patients with colorectal cancer, but is closely associated with the differentiation, TNM stage and distant lymph node metastasis of colorectal cancer.

CTCs are tumor cells shed from the primary tumor into the circulation. The presence of CTCs in the peripheral blood of patients has long been associated with metastasis and poor survival and is now considered an acceptable cancer marker. However, current techniques are limited $(20,21)$. The only commercially available CTC test (CellSearch; Veridex, Raritan, NJ, USA) has a detection rate of $50 \%$ in late-stage patients $(22,23)$. In the present study, we detected CTCs harboring negative enrichment using an immunomagnetic beads method, followed by identification with cytologic analysis, immunofluorescence and imFISH. This combination of methods resulted in detection rates up to $78.57 \%$ in patients with AQP5 overexpression, suggesting that imFISH staining could be used as a detection method for CTCs in future studies. In addition, our study verified that AQP5 overexpression is associated with the possibility of metastasis compared to lower expression of AQP5.

In summary, the AQP5 protein is upregulated in colorectal cancer and is closely related to advanced TNM stage, lymph node metastasis and poor prognosis. AQP5 strong overexpression is highly correlated with gene amplification. Thus, AQP5 may be used as a novel biomarker for colorectal cancer aggressiveness and metastasis.

\section{Acknowledgements}

The authors thank the staff of the Biology and Genetics Laboratory of Xi'an Jiaotong University for their technical assistance in these studies. 


\section{References}

1. American Cancer Society: Cancer facts and figures, 2004. http://acs.org.

2. Kinzler KW and Vogelstein B: Lessons from hereditary colorectal cancer. Cell 87: 159-170, 1996.

3. Koontongkaew S: The tumor microenvironment contribution to development, growth, invasion and metastasis of head and neck squamous cell carcinomas. J Cancer 4: 66-83, 2013.

4. Sekine S, Shimada Y, Nagata T, Moriyama M, Omura T, et al: Prognostic significance of aquaporins in human biliary tract carcinoma. Oncol Rep 27: 1741-1747, 2012.

5. Moon C, Soria JC, Jang SJ, Lee J, Obaidul HM, et al: Involvement of aquaporins in colorectal carcinogenesis. Oncogene 22: 6699-6703, 2003.

6. Wang D and Owler BK: Expression of AQP1 and AQP4 in paediatric brain tumours. J Clin Neurosci 18: 122-127, 2011.

7. Hara-Chikuma $M$ and Verkman AS: Prevention of skin tumorigenesis and impairment of epidermal cell proliferation by targeted aquaporin-3 gene disruption. Mol Cell Biol 28: 326-332, 2008.

8. Burghardt B, Elkaer ML, Kwon TH, Racz GZ, Varga G and Steward MC: Distribution of aquaporin water channels AQP1 and AQP5 in the ductal system of the human pancreas. Gut 52: 1008-1016, 2003.

9. Kang SK, Chae YK, Woo J, Kim MS, Park JC, et al: Role of human aquaporin 5 in colorectal carcinogenesis. Am J Pathol 173: $518-525,2008$

10. Woo J, Lee J, Kim MS, Jang SJ, Sidransky D and Moon C: The effect of aquaporin 5 overexpression on the Ras signaling pathway. Biochem Biophys Res Commun 367: 291-298, 2008.

11. Lee SJ, Chae YS, Kim JG, Kim WW, Jung JH, et al: AQP5 expression predicts survival in patients with early breast cancer. Ann Surg Oncol 21: 375-383, 2014.

12. Shpiz S, Lavrov S and Kalmykova A: Combined RNA/DNA fluorescence in situ hybridization on whole-mount Drosophila ovaries. Methods Mol Biol 1093: 161-169, 2014.
13. McCoy E and Sontheimer H: Expression and function of water channels (aquaporins) in migrating malignant astrocytes. Glia 55: 1034-1043, 2007.

14. Jung HJ, Park JY, Jeon HS and Kwon TH: Aquaporin-5: a marker protein for proliferation and migration of human breast cancer cells. PLoS One 6: e28492, 2011.

15. Zhang Z, Chen Z, Song Y, Zhang P, Hu J and Bai C: Expression of aquaporin 5 increases proliferation and metastasis potential of lung cancer. J Pathol 221: 210-220, 2010.

16. Chen XJ, Yang JH and Zheng W: Effect of topotecan on expression of aquaporin protein 5 and nuclear factor-kappaB in ovarian cancer SKOV3 cells. Ai Zheng 28: 856-860, 2009 (In Chinese).

17. Choi JH, Wu HG, Jung KC, Lee SH and Kwon EK: Apoptosis and expression of AQP5 and TGF-beta in the irradiated rat submandibular gland. Cancer Res Treat 41: 145-154, 2009.

18. Huang YH, Zhou XY, Wang HM, Xu H, Chen J and Lv NH: Aquaporin 5 promotes the proliferation and migration of human gastric carcinoma cells. Tumour Biol 34: 1743-1751, 2013.

19. Wang W, Li Q, Yang T, Bai G, Li D, Li Q and Sun H: Expression of AQP5 and AQP8 in human colorectal carcinoma and their clinical significance. World J Surg Oncol 10: 242, 2012.

20. Onstenk W, Gratama JW, Foekens JA and Sleijfer S: Towards a personalized breast cancer treatment approach guided by circulating tumor cell (CTC) characteristics. Cancer Treat Rev 39: 691-700, 2013.

21. Attard G, Crespo M, Lim AC, Pope L, Zivi A, et al: Reporting the capture efficiency of a filter-based microdevice: a CTC is not a CTC unless it is CD45 negative - letter. Clin Cancer Res 17: 3048-3050, 2011

22. Coumans FA, Ligthart ST, Uhr JW and Terstappen LW: Challenges in the enumeration and phenotyping of CTC. Clin Cancer Res 18: 5711-5718, 2012.

23. Lustberg M, Jatana KR, Zborowski M and Chalmers JJ: Emerging technologies for CTC detection based on depletion of normal cells. Recent Results Cancer Res 195: 97-110, 2012. 